\title{
ON THE CHARACTERIZATION OF SOUSLIN AND BOREL SETS
}

\author{
R.-D.REISS
}

ABSTRACT. Let $X$ be an arbitrary family of sets in the basic set $X$. In this paper Souslin- $X$ sets are represented as projections on $X$ of certain $\sigma \delta$-sets in the cartesian product space of $X$ and the Baire space (or $X$ and the real line). For $L-X$ sets (i.e. Souslin sets defined with disjoint unions; ensembles d'unicité) injective projections are considered. The results also apply to Borel- $\mathfrak{X}$ sets since the system of $L-\mathfrak{x}$ sets includes the Borel- $\mathfrak{X}$ sets under suitable conditions.

1. Introduction. When investigating Borel and Souslin sets as continuous images of some simpler sets one should be aware of the following two results: (a) Each set in a topological space is the continuous, injective image of an open set in a compact Hausdorff space (see [1]). Furthermore, using the discrete topology it is obvious that (b) each set in a topological space is the continuous, injective image of a set being open and closed in a metric space. The problem of finding nontrivial domains is solved here by constructing the topological product of the given space and the Baire space or the real numbers. In this way it is often possible to transfer the topological properties of the given space to the domain of the mapping. As applications of our results, we obtain some well-known theorems which have been proved by techniques different from each other. Our approach reveals that "injective" representations are typical for $L$-sets; the additional settheoretical and topological assumptions which have been used to obtain the corresponding result for Borel sets simply guarantee that the se sets are contained in the system of $L$-sets.

2. Notations and preliminaries. Suppose $X$ is a family of sets. $\mathfrak{X}$ denotes the family of differences of two elements of $X_{\text {. }} \mathfrak{X}_{C^{-}}$(respectively, $x_{\delta}$ ) denotes the family of all countable unions (intersections) of elements of $\mathfrak{X}$. Borel- $X$ is the smallest family which contains $X$ and is closed under differences and countable unions. Let $R$ (respectively, $N$ ) denote the set of all real numbers (nonnegative integers). Let $I=N^{N}$ denote the Baire space and denote by $I(x)=\{a \in I: a \mid n=x\}, x \in N^{n}$, the Baire intervals where $\alpha=\left(\alpha_{i}\right)_{i \in N}$ and $\alpha \mid n=\left(\alpha_{1}, \ldots, a_{n}\right)$. Souslin- $\mathfrak{X}$ is the family of all sets of the form

Received by the editors December $16,1974$.

AMS (MOS) subject classifications (1970). Primary 54H05; Secondary 54 C05.

Key words and phrases. Souslin sets, Borel sets, $\sigma \delta$-sets, injective projections. 


$$
\bigcup_{\alpha \in I} \bigcap_{n \in \mathrm{N}} H(\alpha \mid n)
$$

for some functions $H$ on $\{\alpha \mid n: \alpha \in I, n \in N\}$ to $\mathfrak{X} . L-\mathfrak{X}$ is the family of all sets of the form (2.1) with $\bigcap_{n \in \mathbb{N}}(H(\alpha \mid n) \cap H(\hat{\alpha} \mid n))=\varnothing$ for $\alpha \neq \hat{\alpha}$. It is well known that

(2.2) Souslin-(Souslin-X) $=$ Souslin- $X$,

(2.3) $L \cdot(L \cdot X)=L \cdot X$,

(2.4) Souslin- $X$ is closed under countable unions and countable intersections, and

(2.5) $L-X$ is closed under countable disjoint unions and countable intersections.

For sets $A \subset X \times Y$ let $\Pi_{X}(A)$ denote the projection of $A$ on $X$. $\Pi_{X}(A)$ is called the injective projection of $A$ if $\Pi_{X} \mid A$ is injective.

Suppose $X$ is a topological space. Let $\mathcal{K}(X), \mathcal{F}(X)$, and $\mathcal{G}(X)$ denote the family of compact, closed and open sets in $X$. When no confusion can arise we use $\mathcal{K}, \mathcal{F}$ and $\mathcal{G}$ for these families.

3. The results. In the first part of this section our results are mainly of set-theoretical character. For the sake of reference we include assertion (3.1) which is essentially known (see [5, Theorem 2, p. 343], where the set $B$ is written in a different form).

Hereafter, let $X$ be a family of sets in $X$.

Lemma 1. Let $A \in$ Souslin- $\mathfrak{X}$ be of the form (2.1). Then

(3.1) $\Pi_{X}(B)=A$ for $B=\bigcap_{n \in \mathrm{N}} \bigcup_{x \in N^{n}}(H(x) \times I(x))$.

If we have disjoint summation in (2.1) then, additionally,

(3.2) $\Pi_{X} \mid B$ is injective.

Proof. Because of $\bigcup_{x \in N^{n}}(H(x) \times I(x))=\bigcup_{a \in I}(H(\alpha \mid n) \times\{\alpha\})$, and $(H(\alpha \mid n) \times$ $\{a\}) \cap(H(\hat{\alpha} \mid n) \times\{\hat{\alpha}\})=\varnothing$ for $\alpha \neq \hat{\alpha}$, we obtain

$$
B=\bigcup_{\alpha \in I} \bigcap_{n \in \mathrm{N}}(H(\alpha \mid n) \times\{\alpha\})
$$

Now (3.1) and (3.2) follow in a direct way.

Remark 2. The Baire intervals $I(x), x \in N^{n}$, are open and closed in $I$ and $I(x) \cap I(\hat{x})=\varnothing$ for $x, \hat{x} \in N^{n}$ with $x \neq \hat{x}$. This implies for topological spaces $X$ that

(3.3) $B$ is a closed set in $X \times I$ if $A \in \operatorname{Souslin-F}(X)$, and

(3.4) $B$ is a $\varrho_{\delta}$-set in $X \times I$ if $A \in$ Souslin- $S_{(}(X)$.

To prove (3.3) use the fact that $X \times I$ is the topological sum of the spaces $X \times I(x), x \in N^{n}$, equipped with the relative topology.

(3.3) together with (3.1) yields that a Souslin-F $F(X)$ set is the projection on $X$ of a closed set in the space $X \times I$ (see, e.g., [5, Theorem 5, p. 352]). By (3.2) we know that a $L-\mathcal{F}(X)$ set is an injective projection on 
$X$ of a closed set in $X \times I$. Since $I$ is a complete separable metric space, this proves the converse of a result given by Kunugui [4, Theorems 4 and 7]: For topological spaces $X$ and complete, separable metric spaces $Y$, the (injective) projection on $X$ of a Souslin-F set $(L-\mathcal{F}$ set) in $X \times Y$ is a Souslin-F set $(L-\mathcal{F}$ set) in $X$. (Read, additionally, "injective" when Souslin-F is replaced by $L \cdot \mathcal{F}$. )

The results of Lemma 1 will be reconsidered with the Baire space topologically embedded in the space of the real numbers. As a preparatory step we prove

Lemma 3. Let $I$ be a subset of some set $Y$, and let $M$ be a family of sets in $Y$. Assume that $M$ is closed under finite unions and finite intersec. tions. Let $I \in \mathbb{M}_{\sigma \delta}$ and $I(x) \in\left\{A \cap I: A \in \mathbb{M}_{\sigma}\right\}$ for all $x \in N^{n}$. $n \in N$. Then the set $B$ given in Lemma 1 has the representation

$$
B=\bigcap_{i \in N} \bigcup_{j \in N}\left(H_{i, j} \times H_{i, j}\right)
$$

with $H_{i, j} \in X$ and $M_{i, j} \in \mathbb{M}, i, j \in N$.

Proof. Let $I=\bigcap_{i \in N} T_{i}$ and $I(x)=I \cap S(x)$ for $x \in N^{n}$ where $T_{i}, S(x)$ $\in \mathbb{M}_{\sigma \cdot}$. Then, for each $n \in N$,

$$
\begin{aligned}
\bigcup_{x \in N^{n}}(H(x) \times I(x)) & =\bigcup_{x \in N^{n}}((X \times I) \cap(H(x) \times S(x))) \\
& =(X \times I) \cap \bigcup_{x \in N^{n}}(H(x) \times S(x)) \\
& =\bigcap_{i \in N}\left(\left(X \times T_{i}\right) \cap \bigcup_{x \in N^{n}}(H(x) \times S(x))\right) \\
& =\bigcap_{i \in N} \bigcup_{x \in N^{n}}\left(\left(X \times T_{i}\right) \cap(H(x) \times S(x))\right) \\
& =\bigcap_{i \in N} \bigcup_{x \in N^{n}}\left(H(x) \times\left(T_{i} \cap S(x)\right)\right) .
\end{aligned}
$$

Let $T_{i}=\bigcup_{j \in N} T_{i, j}$ and $S(x)=\bigcup_{j \in N} S_{j}(x)$ with $T_{i, j}, S_{j}(x) \in M$. Then

$$
T_{i} \cap S(x)=\bigcup_{j \in N}\left(\left(\bigcup_{k=1}^{j} T_{i, k}\right) \cap\left(\bigcup_{k=1}^{j} S_{k}(x)\right)\right)
$$

and, hence, $T_{i} \cap S(x) \in \mathbb{M}_{\sigma}$.

This, together with the identity proved above, immediately implies the assertion.

To include Borel sets in our considerations, we prove the following lemma (which extends [3, \$34, II]).

Lemma 4.

(3.5) If $\mathfrak{X} \subset L-X$ and $X \_\subset L-X$, then Borel- $X \subset L-X$. 
(3.6) If $X$ is closed under finite unions and $X_{-} \subset \mathfrak{X}_{\sigma}$, then $\mathfrak{X}_{\sigma} \subset L \cdot \mathfrak{X}_{\text {. }}$

Proof. By Zorn's lemma there exists a maximal system $\pi$ of sets in $L . X$ such that $X \subset \Re_{\text {and }} \pi_{-} \subset L-X$. Applying (2.5), we find, by some standard caluclations, that $\pi$ is closed under differences and countable unions. This proves (3.5).

(3.6) follows in an analogous way as $[2,42,2.2]$.

If $X$ is a complete, separable metric space, then Borel-F $=L-\mathcal{F}$ (see, e.g., [3, pp. 192 and 276]).

Extending a result in [6] (for compact metric spaces and separable metric spaces), we obtain for compact sets:

Proposition 5. Let $X$ be a topological space. If $\mathcal{K}_{-} \subset L_{\cdot} \mathcal{K}$, then $L \cdot \mathcal{K}=$ Borel $\cdot$ K.

Since this proposition is not needed in our further considerations the proof is omitted.

Theorem 6. Let $X$ be a topological space and let $\mathfrak{X} \in\{\mathcal{K}, \mathcal{F}, \mathcal{G}\}$. Each Souslin- $X$ set $\left(L-X\right.$ set) in $X$ is the (injective) projection on $X$ of a $X_{\text {o }}$ set in $X \times R$. Furthermore, if $X_{-} \subset L-X$, then each Borel. $X$ set in $X$ is the injective projection on $X$ of a $X_{\sigma \delta^{-}}$set in $X \times R$.

Proof. Let $\rho$ be a homeomorphism from $I$ to the space $J$ of the irrational numbers (the existence of $\rho$ is well known). Then the Lemmas 1 and 3 hold true for $J$ and $\rho(I(x))$ in place of $I$ and $I(x)$, respectively. Furthermore, $\rho(I(x))$ is open and closed in $J$. Since $J$ is a $\mathcal{G}_{\delta^{-s e t}}$ and a $\mathcal{K}_{\sigma \delta^{-s e t}}$ in $R$, Lemma 3 is applicable with $Y=R$ and $\mathbb{R} \in\{\mathcal{K}, \mathcal{F}, \mathcal{G}\}$ and yields the assertion for Souslin sets and L-sets.

For Borel sets the assertion follows from Lemma 4.

We remark that $R$ can be replaced by $[0,1]$ in Theorem 6 .

For Borel sets and $X=K$, Theorem 6 extends slightly a result given by Sion [7] saying that each Borel-K set in a Hausdorff space with property I (i.e. $\mathcal{K}_{-}\left(\mathcal{K}_{\sigma}\right)$ is the continuous, injective image of a $\mathcal{K}_{\sigma \delta}$-set in a Hausdorff space with property I. Notice that $X \times R$ is a Hausdorff space with property I if this holds for $X$. To prove this apply [7, Theorem 3.5]. By (3.6) we know that $\mathcal{K}_{-} \subset \mathcal{K}_{\sigma}$ implies $\mathcal{K}_{-} \subset L-\mathcal{K}_{\text {. }}$

The following theorem generalizes a result of Hausdorff [3, 334 , IV] namely that a Souslin-F $\mathcal{F}$ set (Borel-F set) in $R^{n}$ is the (injective) projection on $R^{n}$ of a $\biguplus_{\delta}$-set in $R^{n+1}$. Remember that $\varrho_{C} \mathcal{F}_{\sigma}$, e.g., in metric spaces.

Theorem 7. Let $X$ be a topological space such that $\oint_{\mathcal{G}} \mathcal{F}_{\sigma}$. Then each Souslin-F set ( $L-\mathcal{F}$ set, Borel-F set) in $X$ is the (injective) projection of a $\mathfrak{S}_{\delta}$-set in $X \times R$. 
Proof. We shall apply the following identities:

$$
L \cdot \mathscr{G}(X)=L \cdot \mathcal{F}(X)
$$

and

$$
\text { Souslin- }-\mathcal{Y}(X)=\text { Souslin- } \mathscr{F}(X) \text {. }
$$

We shall only prove (3.7) since (3.8) follows similarly. $\varrho_{\mathcal{F}} \mathcal{F}_{\sigma}$ implies $\mathcal{F} \subset \mathcal{S}_{\delta}$. By (2.5), $\mathcal{F} \subset L-\mathcal{G}$. Hence, by (2.3), L.F $\subset$ L $-\mathcal{G}$. Conversely, by (2.5) and (3.6), $\mathcal{G} \subset L \cdot \mathcal{F}$. Hence, by (2.3), L. $\mathcal{G} \subset L \cdot \mathcal{F}$, and (3.7) is proved. (3.7) and (3.8), together with Theorem 6 (with $X=\biguplus_{\text {) }}$ ), immediately imply the assertion.

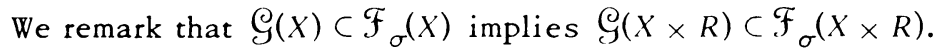

\section{REFERENCES}

1. G. Choquet, Theory of capacities, Ann. Inst. Fourier (Grenoble) 5(1953/54), 131-295. MR 18, 295.

2. H. Hahn, Reelle Finktionen. Teil 1, Academie Verlagsgesellschaft, Leipzig, 1932; reprint, Chelsea, New York, 1948.

3. F. Hausdorff, Mengenlehre, 2nd ed., de Gruyter, Berlin, 1927; English transl., Chelsea, New York, 1957. MR 19, 111.

4. K. Kunugui, La théorie des ensembles analytiques et les espaces abstraits, J. Fac. Sci. Hokkaido Imp. Univ. (1) 4 (1935), 1-40.

5. K. Kuratowski and A. Mostowski, Theory of sets, Monografie Mat., tom 27, PWN, Warsaw, 1966; English transl., North-Holland, Amsterdam; PWN, Warsaw, 1968. MR 34 \#7379; 37 \#5100.

6. W. Sierpiński, Le théorème d'unicité de M. Lusin pour les espaces abstraits, Fund. Math. 21 (1933), 250-275.

7. M. Sion, Continuous images of Borel sets, Proc. Amer. Math. Soc. 12 (1961), 385-391. MR 24 \#A1358.

MATHEMATISCHES INSTITUT, UNIVERSITÄT ZU KÖLN, 5 COLOGNE, WEYERTAL 86, WEST GERMANY 